\title{
Pilot Application of Biofeedback Training Program for Racket Sports Players
}

\author{
${ }^{1}$ Sang-Hyuk Park, ${ }^{2}$ Seunghyun Hwang", ${ }^{\text {SSang-Mi Lee }}$ \\ ${ }^{1}$ Department of Sport Science, Korea Institute of Sport Science, Seoul, South Korea. ${ }^{2}$ Department of \\ Leisure Sport, Kyungpook National University, Sangju, South Korea.
}

Submitted 03 February 2020; Accepted in final form 04 July 2020.

\begin{abstract}
Background. A biofeedback program receives attention as an intervention program for athletes to regulate their psychological state. Objectives. The purpose was to apply for a heart rate variability (HRV) biofeedback training program and validate its effectiveness for racket sports players. Objectives. The purpose was to apply a heart rate variability (HRV) biofeedback training program and validate its effectiveness for racket sports players. Methods. he participants were eight elite sport athletes (three men and five women): five squash players and three tennis players. The HRV biofeedback training program consisted of ten sessions in which the participants performed paced breathing for 10 minutes (e.g., 5-second inspiration and 5-second expiration). The means and standard deviations of the collected data were calculated, and the Wilcoxon signed-rank test was performed using SPSS windows 18.0. Results. Changes in HRV by the biofeedback training were significant. Standard Deviation of Normal to Normal (SDNN; $Z=-2.38, p$ $=0.017)$ in time domain and Low Frequency $(L F, Z=-2.52, p=0.12)$ and the ratio of LF to High Frequency (HF; $Z$ $=-2.52, \mathrm{p}=0.12)$ in frequency domain were increased. Conclusion. The present study provides empirical results needed to expand HRV biofeedback training research for the performance enhancement program of athletes.
\end{abstract}

\section{KEYWORDS: Heart Rate Variability, Biofeedback Training, Sport Performance}

\section{INTRODUCTION}

In elite sport, anxiety and arousal affects performance and judgement competence of an athlete $(1,2)$. Maintaining anxiety and arousal at an appropriate level could be an important psychological strategy for the improvement of intuitive physical reactions and competence for making an effective decision because psychological pressure can cause distractions and performance errors $(3,4)$.

Biofeedback training is used to maintain anxiety and arousal at an appropriate level (5). Biofeedback training simultaneously measures and visualizes physiological parameters such as brainwaves, heart rates, body temperature, respiratory exchange ratio, and heart rate variability (HRV), and increases individuals' self-control of physiological arousal by increasing their awareness of changes in physiological parameters by the changes in the psychological state. Among, various type of biofeedback sources, this study focuses on HRV, which refers to periodic changes between heartbeats, and the changes are controlled by the activation of the sympathetic and parasympathetic nerves of the autonomic nervous system (6). Research and meta-analyses have been conducted on the effectiveness of HRV biofeedback training, that is, the positive effects of the training in terms of reduction of stress and anxiety and improvement in athletic performance $(7,8)$.

HRV biofeedback training induces the synchronization between respiration with a specific frequency and heartbeats, which refers to respiratory sinus arrhythmia (RSA). It is a synchronization phenomenon in which heart rate increase during

*. Corresponding Author:

Seunghyun Hwang, Professor

E-mail: hwangsh@knu.ac.kr 
inspiration and decrease during expiration by the control of the vagus nerve. An individual experiences psychophysiological stability when respiration and heart rates are synchronized $(9,10)$. This is the main purpose of the HRV biofeedback.

Generally, increase in LF indicates a decrease in HRV and increase in anxiety and stress, while an increase in HF indicates an increase in HRV and is related to the reduction of stress. Further, LF/HF indicates the overall balance of the autonomic nervous system, and high LF/HF indicates a high arousal state such as anxiety and tension, while low LF/HF indicates a low arousal state (10).

With the increasing interest of sports psychologists in HRV as a method of psychophysiological analysis for sports performance, research has been conducted on the relationships between competitive state anxiety and HRV (11-13). In addition to correlational studies between the athletes' anxiety and performance, research on HRV biofeedback training for controlling the arousal and anxiety is also being conducted $(2,14$, 15).

In a single case study, Lagos et al. (16) reported an overall increase in HRV and low frequencies (LF) when anxiety and stress were measured using a questionnaire after training a female collegiate golfer with 10 sessions of HRV biofeedback training. Lagos et al. (17) also reported increases in HRV, LF, and high frequencies (HF) after 10 weeks of HRV biofeedback training in another case study for a golfer. Not only focusing on changes in HRV, Mueller, Williams, Haley, and Heick (18) tracked a daily change in HRV of a female triathlete and showed improvement in workout performance and energy by HRV biofeedback. As a group condition, Maman and Kanuoriya (19) confirmed the positive effects of biofeedback training in terms of reducing anxiety and improving performance by recruiting 30 collegiate basketball players as participants and assigning 10 players to the experimental group, which was treated with 10 sessions of HRV breathing biofeedback training, 10 players to the control group, and 10 players to the placebo group, and measured psychological state (trait anxiety, self-efficacy), HRV, and performance (pass, dribble, shoot). Beauchamp et al. (5) conducted a program that supported 20 members of the Canadian national short-track team with continuous biofeedback training for three years and six to ten sessions of a 45 minute HRV biofeedback training program as psychological skills training. The findings of the empirical studies discussed above indicate positive effects of the application of HRV biofeedback training on HRV increase, psychological state evaluations (e.g., competitive state anxiety), and performance in coping with stress in a competitive situation. Recently, Christie, Bertollo, and Werthner (20) applied sensorimotor rhythm neurofeedback training and biofeedback training, and revealed that ice hockey athletes in both feedback trainings showed higher rate of performance improvement in the lab, not for a real shooting performance. This results provide an insight a biofeedback training may show different outcomes due to various contexts.

As Christie et al. (20) suggested, the effect of a biofeedback training on regulating psychological state and performance improvement may reveal different consequences. Therefore, a lot of studies exploring the effectiveness of a biofeedback training should be conducted in order to contribute to standardizing the training protocol. However, no HRV biofeedback training study has applied to elite sport players in Korea. This study would be a cornerstone to initiate more scientific research with respect to the relationship among HRV biofeedback training, psychological state, and sport performance in Korea.

Taken together, the psychological responses to stress and anxiety are controlled by the autonomic nervous system and can be measured and monitored through HRV. Thus, the purpose of this pilot study was to apply a HRV biofeedback training program to the performance enhancement of players of racket sports such as squash and tennis, and to see if HRV biofeedback training could be a psychological skill training to regulate the psychophysiological states for athletic performance. Specifically, changes in players' HRV (i. e., autonomic nervous system balance) before and after their participation in the HRV biofeedback training were examined.

\section{MATERIALS AND METHODS}

Participants. A total of eight athletes (three male and five female players) including five squash players and three tennis players participated in the present study. They participated in a total of 10 sessions (2030 minutes per session, five sessions per week) of the HRV biofeedback training, which was suggested by Lehrer et al. (10). The demographical characteristics of the participants are presented in (Table 1). The average age is 28.37 (SD: 4.02) ranging from 24 to 37 years, and their average career year as elite player is 13.75 (SD: 0.82) ranging from 13 to 15 years. The average heart rate 66.62 (SD: 7.67) ranging from 55 to 80 at resting, which can be considered as a homogeneous group, healthy and young adults with similar athletic career.

Protocol of HRV Training. With an approval of Institutional Review Board, consent to participate in the present study was obtained from coaches and athletes in an introductory session to explain the purpose of HRV biofeedback training. The bio-sensors were attached to participants after they arrived at the laboratory and had a five-minute rest in a comfortable chair. For measuring HRV, a sensor was attached to the tip of the middle finger to measure blood volume pulse (BVP) and a respiration sensor strap, which monitored the expansion and contraction of the abdomen, was worn $5 \mathrm{~cm}$ below the umbilicus to measure respiratory rate. In addition, skin conductance 
sensors (SCS), which measured the arousal state of the body, were attached to the tip of the index and ring fingers to measure electrical skin resistance between the two fingers.

The total number of HRV biofeedback training sessions was determined to be 10 through a meeting between the investigators and experts with reference to the HRV training guidelines developed by Lehrer et al. (10). The training program is described in
(Table 2). Except for the first and tenth session, the participants were instructed to breathe as following a breathing bar on a computer screen (ProComp Infinit-8 Channel Biofeedback System, Thought Technology Ltd., Canada). With the breathing bar, they followed a paced breathing method, in which their ratio in inhale and exhale were equal. They inhaled for five seconds and exhaled for another five seconds.

Table 1. Characteristics of Participants

\begin{tabular}{|c|c|c|c|c|c|c|}
\hline Athlete & Age & Gender & Career & Resting Heart Rate & Number of Session & Sport \\
\hline $\mathbf{A}$ & 32 & Female & 13 & 57 & 10 & Squash \\
\hline B & 24 & Female & 14 & 69 & 10 & Squash \\
\hline C & 26 & Female & 13 & 76 & 10 & Squash \\
\hline D & 30 & Male & 13 & 55 & 10 & Squash \\
\hline $\mathbf{E}$ & 29 & Male & 15 & 65 & 10 & Squash \\
\hline $\mathbf{F}$ & 37 & Male & 15 & 80 & 10 & Tennis \\
\hline G & 25 & Female & 14 & 69 & 10 & Tennis \\
\hline $\mathbf{H}$ & 24 & Female & 13 & 62 & 10 & Tennis \\
\hline
\end{tabular}

Table 2. HRV Biofeedback Training Protocol

\begin{tabular}{|c|c|c|c|c|}
\hline \multicolumn{5}{|c|}{ Program } \\
\hline Session 1 & Sessions 2-4 & Session 5 & Sessions 6-9 & Session 10 \\
\hline $\begin{array}{l}\text { Pre-test and breathe for } \\
5 \text { minutes }\end{array}$ & Breathe for 10 minutes & $\begin{array}{l}\text { Middle-test and breathe } \\
\text { for } 5 \text { minutes }\end{array}$ & Breathe for 10 minutes & $\begin{array}{l}\text { Post-test and breathe for } \\
5 \text { minutes }\end{array}$ \\
\hline
\end{tabular}

Table 3. Descriptive Statistics and Wilcoxon Test of HRV Changes

\begin{tabular}{|c|c|c|c|c|c|}
\hline & n & Pre & Post & $\mathbf{Z}$ & p \\
\hline SDNN & 8 & $73.89 \pm 22.76$ & $116.16 \pm 23.74$ & -2.380 & $0.017 *$ \\
\hline RMSSD & 8 & $64.99 \pm 309.62$ & $86.34 \pm 24.78$ & -1.680 & 0.093 \\
\hline $\mathbf{L F}$ & 8 & $496.09 \pm 320.80$ & $3508.93 \pm 1919.89$ & -2.521 & $0.012 *$ \\
\hline HF & 8 & $531.48 \pm 309.62$ & $676.82 \pm 648.12$ & -.280 & 0.779 \\
\hline LF/HF & 8 & $1.33 \pm 1.17$ & $7.99 \pm 8.00$ & -2.521 & $0.012 *$ \\
\hline Respiratory frequency & 8 & $14.99 \pm 1.20$ & $7.09 \pm 1.81$ & -2.521 & $0.012 *$ \\
\hline
\end{tabular}

Data are presented as mean $\pm \mathrm{SD}, * \mathrm{p}<0.05$

HRV and Respiration Measures. For the time domain of HRV, the root mean square of the successive (NN or RR) differences (RMSSD) and the standard deviation of the entire heartbeat intervals (SDNN) were measured. Subsequently, the HR data were transformed into temporal domain data through spectral analysis, and information in a specific frequency range was used. Low frequencies (LF, 0.04$0.15 \mathrm{~Hz}$ ), high frequencies ( $\mathrm{HF}, 0.15-0.4 \mathrm{~Hz}$ ), and the ratio between the two $(\mathrm{LF} / \mathrm{HF})$ were used for the analysis. In addition to HRV, the respiratory frequency within one minute was measured.

Statistical Analysis. The collected data were analyzed using mean and standard deviation using SPSS Windows 18.0. The Wilcoxon signed-rank test used when the sample size is small (eight in the present study), was performed, and the significance level was set at 0.05 .

\section{RESULTS}

The average values of HRV and respiratory rate of the participants were shown in Table 3. In time domain parameters, the average pre- and post-training SDNN values were $73.89(\mathrm{SD}=22.76)$ and 116.16 $(\mathrm{SD}=23.74)$, respectively. The average pre- and posttraining RMSSD values were $64.99(\mathrm{SD}=309.62)$ and $86.34(\mathrm{SD}=24.78)$, respectively. Although SDNN and
RMSSD were increased after the HRV-biofeedback training, only SDNN showed statistically significant difference. In frequency domain, the pre- and posttraining averages of LF were $496.09(\mathrm{SD}=320.80)$ and 3508.93 ( $\mathrm{SD}=1919.89$ ), and the pre- and post-training averages of HF were $531.48(\mathrm{SD}=309.62)$ and 676.82 $(\mathrm{SD}=648.12)$, respectively. The pre- and post-training $\mathrm{LF} / \mathrm{HF}$ averages were $1.33(\mathrm{SD}=1.17)$ and 7.99 (SD $=8.00)$, respectively. These results show an increase in the post-HRV averages for LF, HF, and LF/HF in the frequency domain; however only LF and LF/HF were significantly increased after the training. Also, a significant decrease was found in respiratory rate.

\section{DISCUSSION}

The effectiveness of HRV biofeedback training for stress, depression, and anxiety has been verified by a number of studies (7), however, research on its relationship with sports performance is still in an early stage. Nonetheless, several empirical studies have validated the application of HRV biofeedback training to the sports field for performance enhancement (8). Accordingly, this pilot study with athletes playing squash and tennis at elite level may contribute to the application of HRV biofeedback training for their psychophysiological stability in the field of sport psychology. Changes in the HRV (autonomic nervous 
system balance and psychophysiological state) of athletes were examined to evaluate improvement in the psychophysiological functioning of athletes.

Significant changes were found in SDNN, LF, $\mathrm{LF} / \mathrm{HF}$, and respiratory rate in the pre- and post-HRV biofeedback. The increase in SDNN in time domain indicates an increase in general HRV after compared to before the HRV biofeedback training. Similarly, Ortega \& Wang (21) found that SDNN in a superior group of shooting athletes was higher than the counterpart. The higher SDNN can be interpreted as psychologically and physiologically better state (10). The increase in LF and LF/HF in frequency domain are associated with increase in arousal state, and it indicates the increased level of focus on biofeedback training (8). Kim, Hwang, Park, Cho, and Kim (22) reported a similar result in LF/HF, which was significant higher during shooting than a resting period of shooting athletes. In a controlled experiment, LF/HF was higher when performing golf putts in front of audience than performing it alone (14). These results indicate that the sympathetic nervous system is relatively more active or that the parasympathetic nervous system is suppressed.

An overall increase of HRV and LF was observed after HRV biofeedback training with female collegiate golfers $(16,17)$. In HRV biofeedback trainings, athletes are instructed to follow a paced breathing by looking at time controlled breathing bar, which may lead psychological load of focusing on the breathing task.

Paced breathing is used to maximize the respiratory sinus arrhythmia (RSA). RSA refers to cardiorespiratory synchronization between respiration and heartbeat patterns, which maintains a pattern of increased heart rate during inhalation and a decrease in heart rate during exhalation $(10,23)$. As the degree of synchronization between breathing and heartbeat is maximized, amygdala and thalamus, which are responsible for emotions such as arousal and fear anxiety, are suppressed, and the parasympathetic nervous system activity is strengthened. (23). To experience psychophysiological stability, the RSA leads the following changes in our body system 1) homeostasis of a baroreceptor located in arterial blood vessels and controlling blood pressure, 2) homeostatic autonomic response reducing sympathetic activity, 3) stimulation of the cholinergic anti-inflammatory system, 4) strengthening of the vagus nerve to regulate the connection of the brain with the area of the brain responsible for emotional regulation, 5) increased gas exchange rate of alveoli through pattern breathing , 6) expansion of the airway through slow breathing, and 7) psychological tranquility as it focuses on breathing (10).

\section{CONCLUSION}

The findings of this pilot study is that HRV biofeedback training contributed to increasing HRV (i.e., SDNN, LF, LF/HF) of racket elite athletes. After HRV biofeedback training, the higher HRV indicates psychologically well-regulated state for athletes. Thus, this conclusion may suggest that HRV biofeedback is a psychological intervention for elite athletes under pressure. Highlighting the psychophysiological benefits, HRV biofeedback training continues to develop as a stress and anxiety management program for psychological performance of elite athletes in the field of sport psychology, which could be supported by the results of this pilot study.

However, there are some limitations. The result was based on the quasi-experimental design with no a control group and limited number of athletes in a racket sports. Thus, the interpretation of the results may be limited to the abovementioned condition, which suggests studies with larger participants in various sports with control groups to receive more empirical data on HRV biofeedback effectiveness. Also, only HRV was measured to evaluate the psychophysiological states, but subjective feeling on the psychological state, such as competitive state anxiety, flow, emotional state, should be measured along with HRV so that dynamic analysis with both approaches on psychological state can be performed for future studies.

\section{APPLICABLE REMARKS}

HRV biofeedback training contributes to change in HRV, which can be an indicator of a psychological state. Thus, HRV biofeedback training is effective as an intervention program for stress and anxiety for elite athletes.

\section{FINANCIAL DISCLOSURE}

No financial sources has been applied to this study.

\section{REFERENCES}

1. Moran AP. The psychology of concentration in sport performers: A cognitive analysis. New York, NY: Routledge; 2016.

2. Mullen R, Hardy L, Tattersall A. The effects of anxiety on motor performance: A test of the conscious processing hypothesis. J Sport Exercise Psychol. 2005;27(2):212-225. doi: 10.1123/jsep.27.2.212

3. Saunders DC, Brissova M, Phillips N, Shrestha S, Walker JT, Aramandla R, et al. Ectonucleoside Triphosphate Diphosphohydrolase-3 Antibody Targets Adult Human Pancreatic beta Cells for In Vitro and In Vivo Analysis. Cell Metab. 2019;29(3):745-754 e744. doi: 10.1016/j.cmet.2018.10.007 pmid: 30449685

4. Whyte J. Rehabilitation. Attention and arousal: basic science aspects. Arch Physic Med Rehabilit. 1992;73(10):940949. doi: 10.5555/uri:pii:000399939290266Y 
5. Beauchamp MK, Harvey RH, Beauchamp PH. An integrated biofeedback and psychological skills training program for Canada's Olympic short-track speedskating team. J Clinic Sport Psychol. 2012;6(1):67-84. doi: 10.1123/jcsp.6.1.67

6. Aubert AE, Seps B, Beckers F. Heart rate variability in athletes. Sports Med. 2003;33(12):889-919. doi: 10.2165/00007256-200333120-00003 pmid: 12974657

7. Goessl VC, Curtiss JE, Hofmann SG. The effect of heart rate variability biofeedback training on stress and anxiety: a meta-analysis. Psychol Med. 2017;47(15):2578-2586. doi: 10.1017/S0033291717001003 pmid: 28478782

8. Jimenez Morgan S, Molina Mora JA. Effect of Heart Rate Variability Biofeedback on Sport Performance, a Systematic Review. Appl Psychophysiol Biofeedback. 2017;42(3):235-245. doi: 10.1007/s10484-017-9364-2 pmid: 28573597

9. Lehrer P. How does heart rate variability biofeedback work? Resonance, the baroreflex, and other mechanisms. Biofeedback. 2013;41(1):26-31. doi: 10.5298/1081-5937-41.1.02

10. Lehrer PM, Gevirtz R. Heart rate variability biofeedback: how and why does it work? Front Psychol. 2014;5:756. doi: 10.3389/fpsyg.2014.00756 pmid: 25101026

11. Blásquez JCC, Font GR, Ortís LC. Heart rate variability and precompetitive anxiety in swimmers. Psicothema. 2009;21(4):531-536.

12. D'Ascenzi F, Alvino F, Natali BM, Cameli M, Palmitesta P, Boschetti G, et al. Precompetitive assessment of heart rate variability in elite female athletes during play offs. Clin Physiol Funct Imaging. 2014;34(3):230-236. doi: 10.1111/cpf.12088 pmid: 24112387

13. Morales J, Garcia V, Garcia-Masso X, Salva P, Escobar R, Busca B. The use of heart rate variability in assessing precompetitive stress in high-standard judo athletes. Int J Sports Med. 2013;34(2):144-151. doi: 10.1055/s-00321323719 pmid: 22972248

14. Murray NP, Raedeke TD. Heart rate variability as an indicator of pre-competitive arousal. Int J Sport Psychol. 2008;39(4):346-355.

15. Neumann DL, Thomas PR. Cardiac and respiratory activity and golf putting performance under attentional focus instructions. Psychol Sport Exercise. 2011;12(4):451-459. doi: 10.1016/j.psychsport.2011.02.002

16. Lagos L, Vaschillo E, Vaschillo B, Lehrer P, Bates M, Pandina R. Heart rate variability biofeedback as a strategy for dealing with competitive anxiety: A case study. Biofeedback. 2008;36(3):109-115.

17. Lagos L, Vaschillo E, Vaschillo B, Lehrer P, Bates M, Pandina R. Virtual reality-assisted heart rate variability biofeedback as a strategy to improve golf performance: a case study. Biofeedback. 2011;39(1):15-20. doi: 10.5298/1081-5937-39.1.11

18. Mueller K, Williams PS, Haley L, Heick J. Heart rate variability biofeedback improves sports performance in an elite female athlete. Cardiopulmonary Physic Therapy J. 2020;31(3):123-132. doi: 10.1097/CPT.0000000000000120

19. Paul M, Garg K. The effect of heart rate variability biofeedback on performance psychology of basketball players. Appl Psychophysiol Biofeedback. 2012;37(2):131-144. doi: 10.1007/s10484-012-9185-2 pmid: 22402913

20. Christie S, Bertollo M, Werthner P. The effect of an integrated neurofeedback and biofeedback training intervention on ice Hockey shooting performance. J Sport Exerc Psychol. 2020:1-14. doi: 10.1123/jsep.2018-0278 pmid: 32005005

21. Ortega E, Wang CJK. Pre-performance physiological state: Heart rate variability as a predictor of shooting performance. Appl Psychophysiol Biofeedback. 2018;43(1):75-85. doi: 10.1007/s10484-017-9386-9 pmid: 29124507

22. Kim Y, Hwang S, Park S, Cho S, Kim. Examining the relation between heart rate variability, flow, and shooting in wheelchair athletes. Exercise Sci. 2019;28(4):339-345. doi: 10.15857/ksep.2019.28.4.339

23. Jerath R, Crawford MW, Barnes VA, Harden K. Self-regulation of breathing as a primary treatment for anxiety. Appl Psychophysiol Biofeedback. 2015;40(2):107-115. doi: 10.1007/s10484-015-9279-8 pmid: 25869930 